\title{
Memory profiles predict dementia over 23-28 years in normal but not successful aging
}

\author{
Maria Josefsson, 1 (1) Anna Sundström, ${ }^{1,2}$ Sara Pudas, ${ }^{3,4}$ Annelie Nordin Adolfsson,, \\ Lars Nyberg, ${ }^{3,4,6}$ and Rolf Adolfsson ${ }^{5}$ \\ ${ }^{1}$ Centre for Demographic and Ageing Research at Umeå University (CEDAR), Umeå, Sweden \\ ${ }^{2}$ Department of Psychology, Umeå University, Umeå, Sweden \\ ${ }^{3}$ Department of Integrative Medical Biology, Umeå University, Umeå, Sweden \\ ${ }^{4}$ Umeå Center for Functional Brain Imaging, Umeå, Sweden \\ ${ }^{5}$ Department of Clinical Sciences, Psychiatry, Umeå University, Umeå, Sweden \\ ${ }^{6}$ Department of Radiation Sciences, Umeå University, Umeå, Sweden
}

Objectives: Prospective studies suggest that memory deficits are detectable decades before clinical symptoms of dementia emerge. However, individual differences in long-term memory trajectories prior to diagnosis need to be further elucidated. The aim of the current study was to investigate long-term dementia and mortality risk for individuals with different memory trajectory profiles in a well-characterized population-based sample.

Methods: 1062 adults (aged 45-80 years) who were non-demented at baseline were followed over 23-28 years. Dementia and mortality risk were studied for three previously classified episodic memory trajectory groups: maintained high performance (Maintainers; 26\%), average decline (Averages; 64\%), and accelerated decline (Decliners; 12\%), using multistate modeling to characterize individuals' transitions from an initial non-demented state, possibly to a state of dementia and/or death.

Results: The memory groups showed considerable intergroup variability in memory profiles, starting 10-15 years prior to dementia diagnosis, and prior to death. A strong relationship between memory trajectory group and dementia risk was found. Specifically, Decliners had more than a fourfold risk of developing dementia compared to Averages. In contrast, Maintainers had a 2.6 times decreased dementia risk compared to Averages, and in addition showed no detectable memory decline prior to dementia diagnosis. A similar pattern of association was found for the memory groups and mortality risk, although only among non-demented.

Conclusion: There was a strong relationship between accelerated memory decline and dementia, further supporting the prognostic value of memory decline. The intergroup differences, however, suggest that mechanisms involved in successful memory aging may delay symptom onset.

Key words: memory decline, episodic memory, death, competing risk, multistate model

\section{Introduction}

Several prospective studies suggest that memory deficits are detectable decades before clinical symptoms of dementia emerge (Boraxbekk et al., 2015; Elias et al., 2000; Rajan et al., 2015). This long preclinical stage provides a critical window for intervention and suggests that thorough neuropsychological assessments are important for early detection. Some studies have observed accelerated decline in multiple cognitive functions in the

Correspondence should be addressed to: Maria Josefsson, Centre for Demographic and Ageing Research, Umeå University, 90187 Umeå, Sweden. Phone: +46 (0)90786 67 78. Email: maria.josefsson@umu.se. Received 02 Jul 2019; revision requested 03 Oct 2019; revised version received 18 Oct 2019; accepted 27 Oct 2019 prodromal phase, starting 3-6 years before clinical symptoms (Albert et al., 2014; Amieva et al., 2004; Cloutier et al., 2015; Li et al., 2017; Wilson et al., 2011). These studies have followed initially cognitively normal individuals longitudinally and examined if an accelerated cognitive rate of change precedes clinical diagnosis. Although previous studies have focused on the nature of memory change in relation to dementia diagnosis, individual differences in longer-term memory trajectories prior to diagnosis need to be further elucidated. Studying the relationship between memory decline and dementia is complicated by the presence of competing events, that is, any event that precludes the event of interest from occurring. For example, in older populations, mortality is a competing event 
to dementia. An underlying assumption in classic survival analysis methods, such as Cox proportional hazards regression, is that death is unrelated to dementia risk. However, dementia and death share many risk factors, thus, the competing risk of death may bias the estimation of the true effects if it is not considered (Chang et al., 2012; Marzona et al., 2016; Prince et al., 2014). The multistate model (MSM) has been developed for the purpose of examine longitudinal time-to-event data of competing events (Austin et al., 2016). While the theory of MSMs has advanced substantially over the last several years, only a few studies are available in cognitive aging research (Marioni et al., 2012; Rouanet et al., 2016). In previous studies of the Betula prospective cohort study (Nilsson et al., 1997), we demonstrated longitudinal evidence suggesting an average reduction in episodic memory after the age of 60 years (Rönnlund et al., 2005). However, there are large interindividual differences (Habib et al., 2007; Josefsson et al., 2012). In the current study, we extend previous work by investigating the association between longitudinal memory profiles, in three previously identified (Josefsson et al., 2012) episodic memory trajectory groups, and the risk for dementia, dementia-free death, or death among demented. We apply a MSM where individuals pass from an initial healthy state, possibly through a dementia state, to the final absorbing state death. In addition, we compare memory performance trajectories with concurrent changes in mini-mental state examination (MMSE) (Folstein et al., 1975) prior to dementia onset and prior to death.

\section{Methods}

\section{The Betula study}

The Betula study is a longitudinal population-based prospective study initiated in 1988, with the objectives to examine health and neurocognitive functions during adult life and old age, and to determine risk factors and preclinical signs of dementia (Nilsson et al., 1997; 2004). Participants considered for the present study were enrolled at the first (1988-1990) and second (1993-1995) wave of data collection, from samples $1(n=1000)$ and 3 $(n=966)$, evenly sex and age distributed over 10 age cohorts ranging from 35 to 85 years of age. Cognitive testing and health assessments have been conducted every 5 years and adjacent to each of the test waves, the occurrence of dementia was determined for each individual. The study was approved by the Regional Ethical Review Board in Umeå and written consent for study participation was obtained from each participant.

\section{Dementia diagnosis assessments}

In the Betula study, presence of dementia has been evaluated adjacent to each test wave, most recently in $2016 / 2017$. Diagnoses were ascertained through a process based on diverse sources of information including reading of medical records from multiple clinical disciplines, substantially from the primary care, medicine, neurology, and geriatric clinics within the county. Thus, other clinicians' assessments, information on medical and social status, medication history, results from available neuroradiological examinations, and evident clinical signs of neurocognitive impairment were integrated in the assessment of determining possible dementia. For the study participants participating in the health and cognitive test assessments, the dementia evaluation also included collateral information from the neuropsychological testing and health examinations. Dementia was not diagnosed unless the disease was characterized by a progressive course. Moreover, for study participants participating in the health and cognitive test assessments, the dementia evaluation also included the test staff's observations, significant drop in performance/low test results on cognitive tests and MMSE scores, as well as the study participants' subjective perception of memory failure and/or concern of dementia.

The described diagnostic procedure was performed for each participant at baseline to rule out presence of dementia, and every 5 years in order to follow the expected progressive course of previously ascertained diagnosis, and furthermore to identify new cases with dementia and determine the year of disease onset. Disease onset was defined as the time at which an individual fulfilled the core criteria for dementia, that is, when the clinical symptoms became sufficiently severe to interfere with social functioning and instrumental activities of daily living (McKhann et al., 2011). The same research geropsychiatrist (co-author R. A.) coordinated the diagnostic evaluation throughout the study period and was responsible for the final diagnoses, which were defined according to the Diagnostic and Statistical Manual of Mental Disorders, 4th edition (DSM-IV) criteria (American Psychiatric Association, 2000).

\section{Cognitive measures}

For the present study, two assessments were used to characterize cognitive function. First, a composite episodic memory score (EMS). The sum of scores on five different episodic memory tasks: (1) immediate free recall of 16 visually and orally presented short sentences, (2) delayed cued recall of nouns from the previously presented sentences, (3) immediate free recall of 16 enacted sentences, (4) delayed cued recall of nouns from the enacted sentences, and 
A

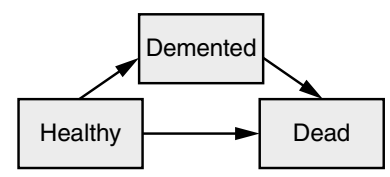

B

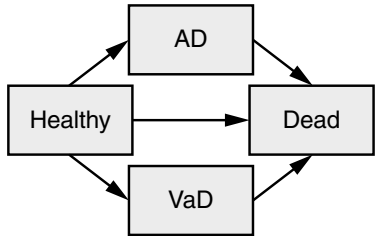

Figure 1. Graphical representation of the multistate models. (Panel A) The illness-death model. (Panel B) Each subject starts in the healthy state. From there, they can possibly either be diagnosed with Alzheimer's (AD) or vascular dementia (VaD), or die. A subject first diagnosed with dementia, they can also transition to death.

(5) immediate free recall of a list of 12 orally presented nouns (details are found in Nilsson et al., 1997). The EMS score could range from 0 (minimum) to 76 (maximum). Second, MMSE (Folstein, Folstein and McHugh, 1975), a screening instrument commonly used to assess general cognitive dysfunction.

\section{Memory trajectory groups}

The 1558 participants from the original Betula samples S1 and S3, who completed two or more test sessions, were previously classified as having maintained high episodic memory performance ("Maintainers"; 18\%), average decline ("Averages"; $68 \%$ ), or accelerated decline ("Decliners"; $13 \%$ ). The model was based on their performance on up to five repeated EMS over 15-20 years and corrected for dropout. Individuals were classified as having episodic memory decline/maintenance if their rates of change fell below/above 1 standard deviation (SD) of a model prediction that accounted for their age and individual baseline performance (Josefsson et al., 2012; Pudas et al., 2013). For individuals diagnosed with dementia, only EMS prior to diagnosis were considered.

\section{APOE genotyping}

Apolipoprotein E (APOE) genotypes were determined by a polymerase chain reaction. For details of the procedure, see Josefsson et al. (2017).

\section{Statistical analysis}

We used linear mixed models (Fitzmaurice et al., 2008) to analyze the association between the memory groups and 15-20 years memory change prior to dementia diagnosis or death. Fixed-effect terms included an overall intercept, indicator variables for the decline and maintained-memory groups, plus interactions with time measured in years to diagnosis or death. For the non-demented and non-deceased group, the end point was considered the last test occasion at the fifth wave. In addition, age, age-squared and an indicator for the first testing occasion were included as a fixed effect to account for practice effects. The model also included a subject-specific random intercept, to account for within-subject correlations between repeated measurements. To characterize the association between dementia, mortality, and memory trajectory groups, we adopted a MSM using age as the timescale. In the present study, we considered the two MSMs that are illustrated in Figure 1. First, the illness-death model in which each subject starts in the healthy state and depending on event, transition to either a state of dementia, considering both Alzheimer's disease (AD) and vascular dementia $(\mathrm{VaD})$, or to the final absorbing state death. If a subject is first diagnosed with dementia, they can also transition to the final state death. In the second model, $\mathrm{AD}$ and $\mathrm{VaD}$ were treated as two separate states as preliminary analyses indicated that there were some differences in memory trajectories and predictors between the groups. For each permitted transition in the MSM, there is a corresponding time-to-event model, with cause-specific hazard rates (HR). Subject-specific characteristics included in the model are: binary indicator variables for Maintainers and Decliners, sex, carrier of the APOE $\varepsilon 4$ allele, and low education level, corresponding to below median years of education. We employ a semi-parametric MSM where the hazards are assumed to be proportional and each transition has its own baseline hazard and specific covariate effects. Preliminary analysis revealed a violation of the proportional hazards assumption for the Maintainer variable. To overcome this problem, we performed median-split age-stratified analyses. Considering an old age cohort and an old-old age cohort, below 87 years of age and 87 or older, respectively, at the study end. An alpha level of 0.05 was considered statistically significant. Analyses were carried out using R (R Core Team, 2017). Mixed models were fitted using the lme 4 package. $p$-values were calculated using Satterthwaite's approximation (Goodnight, 1978) implemented in the lmerTest package. The mstate package was used to fit the MSMs to the data.

\section{Study sample}

The inclusion criteria for the present study were (1) completing the episodic memory testing at two or more test sessions, in order to have been classified into a memory trajectory group, (2) undergoing APOE genotyping, and (3) the expected age at the study end to be 70 or older. The oldest cohort $(n=14)$, aged 85 years at enrollment, was excluded to avoid healthy survivor bias. Further, participants with other dementia diagnoses than $\mathrm{AD}$ 
Table 1. Study participants demographic, cognitive, and health data across memory groups. $n(\%)$, mean \pm standard deviation

\begin{tabular}{lcccr}
\hline & $\begin{array}{c}\text { DECLINERS } \\
(n=123)\end{array}$ & $\begin{array}{c}\text { AVERAGES } \\
(n=683)\end{array}$ & $\begin{array}{c}\text { MAINTAINERS } \\
(n=256)\end{array}$ & $p$ \\
Age at enrollment & $61.4 \pm 9.8$ & $60.9 \pm 10.1$ & $63.2 \pm 9.5$ & $<0.001$ \\
Females & $63(51.2 \%)$ & $352(51.5 \%)$ & $166(64.8 \%)$ & 0.011 \\
Education, years & $8.5 \pm 2.8$ & $9.0 \pm 3.5$ & $10.2 \pm 4.3$ & $<0.001$ \\
Apoe $\varepsilon 4$ carriers & $50(40.7 \%)$ & $196(28.7 \%)$ & $66(25.8 \%)$ & 0.010 \\
$\mathrm{AD}$ & $32(26.0 \%)$ & $68(10.0 \%)$ & $13(5.1 \%)$ & $<0.001$ \\
VaD & $23(18.7 \%)$ & $46(6.7 \%)$ & $6(2.3 \%)$ & $<0.001$ \\
Deceased non-demented & $65(52.9 \%)$ & $309(45.3 \%)$ & $122(47.7 \%)$ & 0.456 \\
Deceased, demented & $45(81.8 \%)$ & $81(71.1 \%)$ & $15(78.9 \%)$ & 0.996 \\
\hline
\end{tabular}

Abbreviations: $\mathrm{AD}$, Alzheimer's disease; $\mathrm{VaD}$, vascular dementia.

and $\mathrm{VaD}$ were excluded. Specifically, Lewy body dementia $(n=6)$, dementia due to Parkinson's disease $(n=5)$, dementia not other-wise specified $(n=14)$, frontotemporal dementia $(n=2)$, and progressive supranuclear paralysis $(n=1)$.

\section{Results}

\section{Study population}

A total of 1062 participants met the inclusion criteria for this study, $55 \%$ of whom were females. The mean age of participants at enrollment was 61.5 years (SD 10.0). During the follow-up period, 188 individuals developed dementia (AD, $n=113$ and $\mathrm{VaD}, n=75), 407$ died without dementia diagnosis, and among those who developed dementia $141(75 \%)$ died during the study period (AD, $n=77$ and $\mathrm{VaD}, n=64$ ). Remaining 467 individuals were non-demented/non-deceased at the study end point. Of the included participants, 683 participants (64\%) were previously classified (Josefsson et al., 2012) as having an average episodic memory decline (Averages), $256(26 \%)$ as having maintained memory performance (Maintainers), and 123 (12\%) as having accelerated memory decline (Decliners). The mean age at onset of dementia was 81.5 years for Averages, whereas Decliners were 4.0 years younger $(p<0.001)$, and Maintainers were 4.1 years older $(p=0.001)$. For the deceased, non-demented group, median age at event of death was 81.0 years for Averages, Decliners were 2.7 years younger $(p=0.088)$, and Maintainers were 2.8 years older $(p=0.004)$. There was no significant difference in proportions of Maintainers, Averages, and Decliners between $\mathrm{AD}$ and $\mathrm{VaD}(p=0.725)$, and no significant differences for the memory groups in age at onset between $\mathrm{AD}$ and $\mathrm{VaD}$. Thus, having a more beneficial memory trajectory was consistently associated with better outcomes in terms of older age at disease onset or death. The characteristics of the analytic sample, divided by memory groups, are shown in Table 1.

\section{Memory trajectories prior to dementia onset or death}

The study participants were divided into four groups according to their follow-up status, (1) $\mathrm{AD}$ group; individuals who developed $\mathrm{AD}$ within the studied period, (2) $\mathrm{VaD}$ group; individuals who developed $\mathrm{VaD}$ within the study period, (3) A group of deceased individuals who died without the presence of dementia during the studied period, and (4) Healthy group; non-demented and nondeceased individuals at the study end point in 2016. A set of linear mixed models were further fitted to assess memory trajectory profiles, in EMS and MMSE, for each of the four groups. The memory trajectory groups showed only minor discrepancies in MMSE over time. That is, similar performance up to less than 5 years prior to onset or death, and nonsignificant memory group differences in decline. However, in stark contrast the results for EMS revealed considerable inter-individual variability in cognitive profiles prior to dementia onset, for both $\mathrm{AD}$ and $\mathrm{VaD}$, and prior to death (see Figure 2). Notably, Maintainers had a high, stable memory performance prior to $\mathrm{AD}$ diagnosis and prior to death. Prior to $\mathrm{VaD}$ diagnosis, the Maintainer group revealed an incipient decline. Both Averages and Decliners revealed a decline in EMS starting 10-15 years prior to dementia diagnosis or event of death, where Decliners showed an accelerated decline compared to Averages. The rates of change did not differ significantly for $\mathrm{AD}$ and $\mathrm{VaD}$ across the cognitive profiles. In the healthy group, Averages and Decliners revealed a decline in memory performance over time, Averages although at a higher level and showing less steep decline. In comparison, Maintainers revealed a stable performance over time. 
A

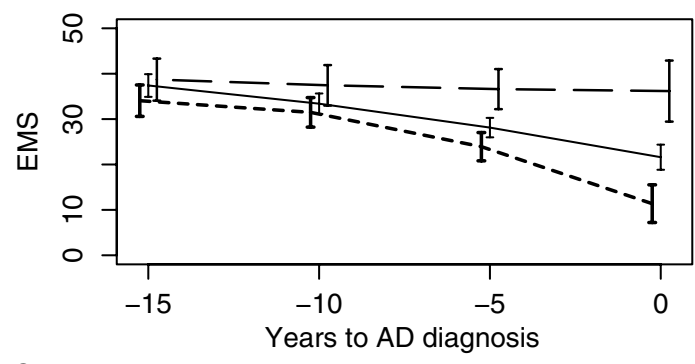

C

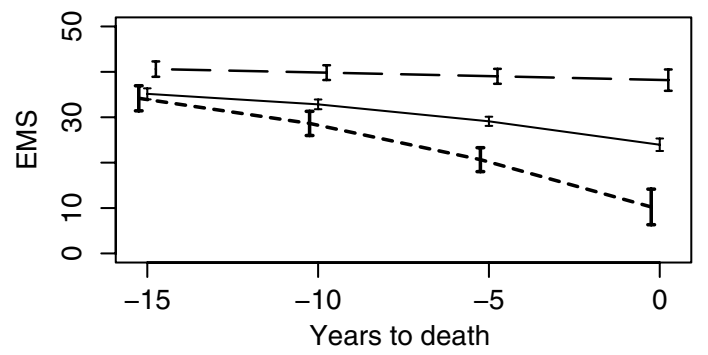

B

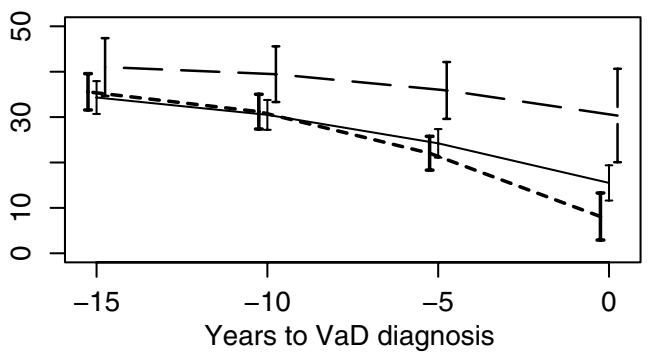

$\mathrm{D}$

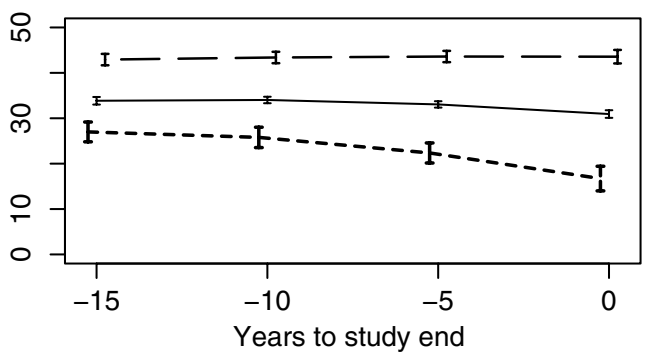

Averages

- Maintainers

- Decliners

Figure 2. Predicted episodic memory score (EMS) trajectories stratified by memory groups. Parameters are derived from mixed models after controlling for age and practice effects. (Panel A) Dementia, AD: individuals who developed AD within the studied period. (Panel B) Dementia, VaD: individuals who developed VaD within the studied period. (Panel C) Dementia-free deceased group: non-demented individuals who died during the studied period. (Panel D) Healthy: non-demented and non-deceased individuals at the last test wave.

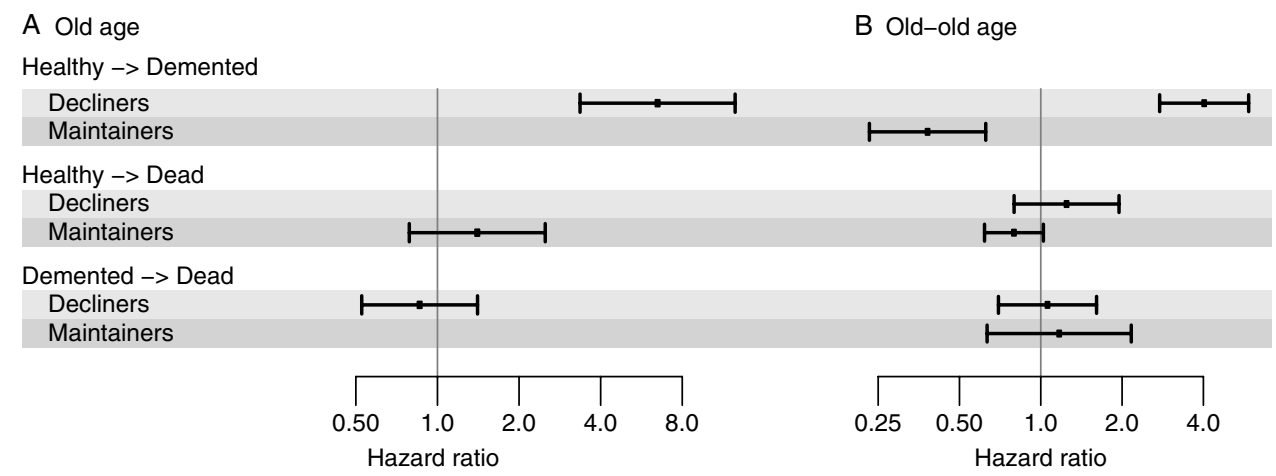

Figure 3. Hazard rates (HR) and $95 \%$ confidence intervals ( $95 \% \mathrm{Cls}$ ) from the multistate model for transitions from a healthy state to a demented and further to a deceased state, or a dementia-free deceased state. (Panel A) Old cohort and (Panel B) Old-old cohort.

\section{Multistate model}

We used a MSM to investigate differences in the progression to dementia and/or death for the memory groups. Two sets of models were fitted; first a model considering dementia (comprising $\mathrm{AD}$ and $\mathrm{VaD}$ together), and a second model considering $\mathrm{AD}$ and $\mathrm{VaD}$ separately. Stratified analyses were conducted for an old, 71-86 years of age at the study end $(n=519)$, and an old-old cohort, $87-107$ years of age $(n=543)$. In the old cohort, $74 \%$ were non-demented and alive at the study end among Averages, as compared to $81 \%$ among Maintainers and $51 \%$ among Decliners. In the old-old cohort, corresponding numbers were $15 \%$ among Averages, 23\% among Maintainers, and 3\% among Decliners. The results for the first model (comprising $\mathrm{AD}$ and $\mathrm{VaD}$ together) are shown in Figure 3. Specifically, in the old cohort, Decliners had a 6.5 times increased risk (95\% confidence interval (CI); 3.02-14.40) of developing dementia compared to Averages. Notably, $n=0$ of the Maintainers developed dementia. In the old-old cohort, similar patterns were shown. That is, Decliners had a 4.0 times increased risk (95\% CI; 2.75-5.88) of developing dementia compared to Averages. Maintainers had a 2.6 times decreased risk $(\mathrm{HR}=0.38,95 \% \mathrm{CI}$; 0.23-0.63) of developing dementia compared to Averages. For the transition to a dementia-free death in both the old and the old-old cohort all effects were nonsignificant, 
Decliners; $\mathrm{HR}=1.4,95 \% \mathrm{CI} ; 0.79-2.50$ in the old cohort, and $\mathrm{HR}=1.2,95 \% \mathrm{CI} ; 0.53-1.41$ in the old-old cohort, and Maintainers; HR $=0.86,95 \%$ CI; $0.53-1.41$ in the old cohort and $\mathrm{HR}=0.8,95 \%$ CI; $0.62-1.02$ in the old-old cohort. Further, for all transitions from dementia to a deceased state, the memory trajectory groups had small and nonsignificant effects. Thus, memory group was not significantly associated with mortality. To investigate if the results differed for $\mathrm{AD}$ and $\mathrm{VaD}$ subgroups, we modeled these groups separately. The results in the old cohort revealed that Decliners had a 6.6 times increased risk (95\% CI; 3.02-14.40) of developing $\mathrm{AD}$ compared to Averages, and a 6.2 times increased risk (95\% CI; 1.83-21.31) of developing $\mathrm{VaD}$. In the old-old cohort, similar patterns were shown. That is, Decliners had a 3.7 times increased risk (95\% CI; 2.19-6.15) of developing $\mathrm{AD}$ compared to Averages, and a 4.5 times increased risk (95\% CI; 2.56-7.87) of developing $\mathrm{VaD}$. Maintainers had a 2.3 times decreased risk $(\mathrm{HR}=0.44,95 \% \mathrm{CI} ; 0.24-0.81)$ of developing $\mathrm{AD}$ compared to Averages, while the risk of developing $\mathrm{VaD}$ was 3.4 times decreased $(\mathrm{HR}=0.29,95 \% \mathrm{CI} ; 0.12-0.70)$. For the transition from a $\mathrm{VaD}$ to a deceased state, Maintainers had a 2.6 times increased risk of death (95\% CI; 0.92-7.07) in the old-old cohort. For all other transitions to a deceased state, the memory trajectory groups had small and nonsignificant effects. Thus, only small differences were found for $\mathrm{AD}$ and $\mathrm{VaD}$. To further investigate if our findings were affected by other characteristics, we considered a model including the memory groups, as well as, sex, education, and APOE $\varepsilon 4$ as predictor variables. For the memory groups, similar results were found as for the second model (treating $\mathrm{AD}$ and $\mathrm{VaD}$ separately) for the transition to either $\mathrm{AD}$ or $\mathrm{VaD}$ state. In addition, for the transition from a $\mathrm{VaD}$ to deceased state, Maintainers had a 3.0 times increased risk of death (95\% CI; 1.01-8.72) in the old-old cohort. All other transitions were small and nonsignificant for the memory groups. APOE $\varepsilon 4$ carriers had a 9.0 times increased risk (95\% CI; 3.39-24.14) in the old cohort and 3.0 increased risk (95\% CI; 1.94-4.59) in the old-old cohort of developing AD compared to noncarriers. The APOE genotype was not significantly associated with any other transitions. Female sex was associated with a 2.8 times (95\% CI; $1.14-2.59$ ) and 2.0 times (95\% CI; 1.37-2.25) increased risk of developing $\mathrm{AD}$ in the old and old-old cohort, respectively. In the old cohort, male sex conferred a significant 1.7 times increased risk of death without dementia (95\% CI; 1.14-2.54) and in the old-old cohort a 2.0 times increased risk $(95 \% \mathrm{CI}$; 1.58-2.51). Hence, among non-demented men died at a younger age than females. For the transition to dementia death, gender had small and nonsignificant effects. The absence of association between the memory groups and death was somewhat surprising. Thus, in a follow-up analysis, we analyzed if similar patterns were found for the subset of only nondemented individuals (through the studied period) using ordinary Cox regression. The results showed a significant association between the memory groups and death only in the old-old cohort, Decliners; $\mathrm{HR}=1.6,95 \% \mathrm{CI} ; 0.90-2.86$, in the old cohort, and $\mathrm{HR}=2.03,95 \% \mathrm{CI} ; 1.30-3.16$, in the old-old cohort, Maintainers; HR $=0.82,95 \% \mathrm{CI}$; 0.50-1.34 in the old cohort, and HR $=0.73,95 \%$ CI; 0.57-0.93 in the old-old cohort.

\section{Discussion}

This population-based study investigated long-term dementia outcomes for three memory trajectory groups (Maintainers, Averages, and Decliners) in a well-characterized population-based sample of older adults who were non-demented at baseline. The main finding was a strong relationship between longitudinal change in episodic memory performance and dementia risk. For those who developed dementia, this could generally be traced in cognitive patterns $10-15$ years before clinical diagnosis. In contrast, only little decline was observed in MMSE during the same period, suggesting that broad screening measures, such as MMSE, lack the sensitivity to detect or follow the course of the preclinical phase of dementia. Importantly, the results highlight the heterogeneity of cognitive trajectories in older adults, and a significant proportion of elderly showed evidence of successful cognitive aging, which is consistent with findings from past studies (Hayden et al., 2014; Muniz Terrera et al., 2010; Nyberg and Pudas, 2018; Zahodne et al., 2015). Furthermore, the Maintainers showed virtually no signs of memory impairment as individuals' progress toward dementia, suggesting that the absence of quantifiable cognitive decline does not necessarily mean protection against dementia. These results are in line with the theory of cognitive reserve (Stern, 2002). Specifically, cognitive reserve is thought to delay the onset of symptoms despite accumulating pathology, with a more rapid decline once pathology reaches a critical threshold. In the current study, individuals in the Maintainers group also had a higher education level, which is the most common proxy for cognitive reserve, than Decliners and Averages, further supporting the theory. Another complementary theory suggests that brain maintenance postpones brain pathology, rather than coping with its presence (Nyberg et al., 2012). Nevertheless, 
more research is needed to confirm and further characterize distinguishing characteristics of this high-performing group. In contrast, the Decliner group revealed an accelerated decline in cognitive function starting a decade before dementia onset. Notably, in the old-old cohort, only a small proportion $(3 \%)$ in the group with accelerated decline was non-demented and alive at the study end. These results highlight the strong predictive relationship between cognitive decline and unfavorable aging outcomes. These findings, based on unusually long follow-up, confirm that accelerated episodic memory impairment provides an early indication of dementia and shed light on the time course of impairment. In addition, as expected, the Decliners were significantly more often APOE $\varepsilon 4$ carriers and had lower education level, suggesting these factors modulates individuals' memory trajectories in their progression to dementia, which is in line with previous studies (Amieva et al., 2004; Chang et al., 2012). This study is innovative in its design in that it uses a multistate modeling approach that gives a more precise and complete picture of the course of the disease. A surprising finding was that episodic memory decline was not a risk factor for the progression to death. This may be explained by that the MSM approach considers all individuals, demented as well as non-demented prior to onset, as opposed to ordinary Cox regression where usually only a subset of individuals who remain non-demented throughout the study period is considered. In a follow-up analysis of only nondemented individuals, we found an association between the memory groups and the risk of death, which are in line with previous studies (e.g. Laukka et al., 2006; Laukka et al., 2008; Thorvaldsson et al., 2008). These results suggest that accelerated terminal decline is driven by non-demented individuals and that episodic memory decline is less prognostic for mortality among demented, who are all already affected with more advanced forms of decline. We do, however, observe large heterogeneity in terminal decline trajectories among non-demented, that is, similar heterogeneity in trajectory patterns that were observed prior to dementia onset was also observed in the time preceding dementia-free death. These findings are in line with previous work suggesting that person-specific factors such as genetics and comorbidity may contribute to differences in trajectories prior to death (Wilson et al., 2007; Fabbri et al., 2016; Gerstorf and Ram, 2013). We extend previous studies of competing risks for dementia and death (Li et al., 2017; Prince et al., 2014) by studying two types of dementia disorders, $\mathrm{AD}$ and $\mathrm{VaD}$. Although, similar associations were found between the memory groups and $\mathrm{AD}$ or $\mathrm{VaD}$ risk, the predictors of $\mathrm{AD}$ and $\mathrm{VaD}$ were different, where female gender and APOE $\varepsilon 4$ were risk factors for $\mathrm{AD}$ but not for $\mathrm{VaD}$. Our results demonstrate that there are marked similarities in memory profiles between the disorders along with some differences with regard to strong risk factors, which is in line with previous studies (Karantzoulis and Galvin, 2011; Looi and Sachdev, 1999; Pilon et al., 2016). Thus, a compound analysis may bias the estimation of true covariate effects. Moreover, in this study, we saw a somewhat higher prevalence of $\mathrm{VaD}(7.1 \%)$ than usually is observed in populationbased studies. The population in northern Sweden is characterized by high serum cholesterol levels, probably reflecting a higher intake of saturated fat, and a high consumption of smokeless tobacco. These factors may explain a substantial part of the increased risk in coronary heart disease morbidity and mortality found in previous studies (Huhtasaari et al., 1988), and is a possible explanation for higher prevalence of $\mathrm{VaD}$ in this cohort.

In this study, diagnoses of dementia were ascertained through reading of medical records from multiple clinical disciplines. The diagnostic assessment also included collateral information from the health and cognitive assessments and predefined criteria for identifying individuals with potential increased risk of dementia. The method used has its limitations and may lead to an underestimation of the number of demented individuals, especially mild cases, and individuals with good cognitive reserve. However, diagnoses were evaluated at repeated occasions in order to establish a reliable dementia status and time of onset, minimizing the risk of misclassification. Importantly, before these results might have clinical relevance, it is necessary to evaluate the predictive performance of episodic memory decline in a clinical setting.

\section{Conflicts of interest}

The authors declare no conflicts of interests.

\section{Funding}

Financial support was provided through a regional agreement between Umeå University and Västerbotten County Council (ALF).

\section{Description of authors' roles}

Study conception and design: MJ, LN, RA. Dementia diagnostics: RA. Data analysis interpretation of data: MJ, AS, SP. Drafting of manuscript: MJ, AS, SP, ANA. Critical revision: MJ, AS, SP, ANA, LN, RA. 


\section{Acknowledgments}

This work was supported by the Swedish Research Council for Health, Working Life and Welfare, and is part of the program Paths to Healthy and Active Ageing (Dnr 2013 - 2056). The Betula Project is supported by Knut and Alice Wallenberg foundation (KAW) and the Swedish Research Council (K2010-61X-21446-01).

\section{References}

Albert, M. et al. (2014). Cognitive changes preceding clinical symptom onset of mild cognitive impairment and relationship to ApoE genotype. Current Alzheimer Research, 11, 773-784.

American Psychiatric Association. (2000). Diagnostic and Statistical Manual of Mental Disorders-IV-TR. Washington, DC: American Psychiatric Association.

Amieva, H. et al. (2004). Annual rate and predictors of conversion to dementia in subjects presenting mild cognitive impairment criteria defined according to a populationbased study. Dementia and Geriatric Cognitive Disorders, 18, 87-93.

Austin, P.C., Lee, D. and Fine, J.P. (2016). Introduction to the analysis of survival data in the presence of competing risks. Circulation, 133, 601-609.

Boraxbekk, C.J., Lundquist, A., Nordin, S., Nyberg, L., Nilsson, L.G. and Adolfsson, R. (2015). Free recall episodic memory performance predicts dementia ten years prior to clinical diagnosis, findings from the Betula longitudinal study. Dementia and Geriatric Cognitive Disorders Extra, 5, 191-202.

Chang, C.-C.H., Zhao, Y., Lee, C.-W. and Ganguli, $M$. (2012). Smoking, death, and Alzheimer's disease: a case of competing risks. Alzheimer Disease and Associated Disorders, 26,300 .

Cloutier, S., Chertkow, H., Kergoat, M.J., Gauthier, S. and Belleville, S. (2015). Patterns of cognitive decline prior to dementia in persons with mild cognitive impairment. Fournal of Alzheimer Disease, 47, 901-913.

Elias, M.F., Beiser, A., Wolf, P.A., Au, R., White, R.F. and D'agostino, R.B. (2000). The preclinical phase of Alzheimer disease: a 22-year prospective study of the Framingham Cohort. Archives of Neurology, 57, 808-813.

Fabbri, E. et al. (2016). Association of accelerated multi-morbidity and age-related cognitive decline in older, non-demented participants from the Baltimore longitudinal study of aging. Fournal of the American Geriatrics Society, 64, 965-972.

Fitzmaurice, G., Davidian, M., Verbeke, G. and Molenberghs, G. (2008). Longitudinal Data Analysis. Boca Raton, FL: Chapman \& Hall/CRC.

Folstein, M.F., Folstein, S.E. and McHugh, P.R. (1975). "Mini-mental state": a practical method for grading the cognitive state of patients for the clinician. Fournal of Psychiatric Research, 12, 189-198.

Gerstorf, D. and Ram, N. (2013). Inquiry into terminal decline: five objectives for future study. Gerontologist, 53, 727-737.
Goodnight, J.H. (1978). Tests of Hypotheses in Fixed-Effects Linear Models. SAS Technical Report R-101. Cary, NC: SAS Institute Inc.

Habib, R., Nyberg, L. and Nilsson, L.-G. (2007). Cognitive and non-cognitive factors contributing to the longitudinal identification of successful older adults in the Betula study. Aging Neuropsychology and Cognition, 14, 257-273.

Hayden, K M. et al. (2014). Pre-clinical cognitive phenotypes for Alzheimer disease: a latent profile approach. The American fournal of Geriatric Psychiatry, 22, 1364-1374.

Huhtasaari, F., Asplund, K. and Wester, P.O. (1988). Cardiovascular risk factors in the Northern Sweden MONICA Study. Acta Medica Scandinavica, 224, 99-108.

Josefsson, M., de Luna, X., Pudas, S., Nilsson, L.-G. and Nyberg, L. (2012). Genetic and lifestyle predictors of 15-year longitudinal change in episodic memory. Fournal of the American Geriatrics Society, 60, 2308-2312.

Josefsson, M., Larsson, M., Nordin, S., Adolfsson, R. and Olofsson, J. (2017). APOE- 44 effects on longitudinal decline in olfactory and non-olfactory cognitive abilities in middle-aged and old adults. Scientific Reports, 7, 1286.

Karantzoulis, S. and Galvin, J.E. (2011). Distinguishing Alzheimer's disease from other major forms of dementia. Expert Review of Neurotherapeutics, 11, 1579-1591.

Laukka, E.J., MacDonald, S.W. and Bäckman, L. (2006). Contrasting cognitive trajectories of impending death and preclinical dementia in the very old. Neurology, 66, 833-838.

Laukka, E.J., MacDonald, S.W. and Bäckman, L. (2008). Terminal-decline effects for select cognitive tasks after controlling for preclinical dementia. American fournal of Geriatric Psychiatry, 16, 355-365.

Li, G. et al. (2017). Cognitive trajectory changes over 20 years before Dementia diagnosis: a large cohort study. Fournal of the American Geriatrics Society, 65, 2627-2633.

Looi, J.C. and Sachdev, P.S. (1999). Differentiation of vascular dementia from $\mathrm{AD}$ on neuropsychological tests. Neurology, 53, 670-670.

Marioni, R.E., Valenzuela, M.J., Van den Hout, A., Brayne, C. and Matthews, F.E. (2012). Active cognitive lifestyle is associated with positive cognitive health transitions and compression of morbidity from age sixty-five. PLoS One, 7, e50940.

Marzona, I. et al. (2016). Risk of dementia and death in patients with atrial fibrillation: a competing risk analysis of a population-based cohort. International fournal of Cardiology, 220, 440-444.

McKhann, G M. et al. (2011). The diagnosis of dementia due to Alzheimer's disease: recommendations from the National Institute on Aging Alzheimer's Association workgroups on diagnostics guidelines for Alzheimer's disease. Alzheimer's E Dementia, 7, 263-269.

Muniz Terrera, G., Brayne, C., Matthews, F. and the CC75C Study Collaboration Group. (2010). One size fits all? Why we need more sophisticated analytical methods in the explanation of trajectories of cognition in older age and their potential risk factors. International Psychogeriatrics, 22, 291-299. 
Nilsson, L.-G. et al. (1997). The Betula prospective cohort study: memory, health, and aging. Aging Neuropsychology and Cognition, 4, 1-32.

Nilsson, L.-G., Adolfsson, R., Bäckman, L., de Frias, C.M., Molander, B. and Nyberg, L. (2004). Betula: a prospective cohort study on memory, health and aging. Aging Neuropsychology and Cognition, 11, 134-148.

Nyberg, L., Lövdén, M., Riklund, K., Lindenberger, U. and Bäckman, L. (2012). Memory aging and brain maintenance. Trends in Cognitive Science, 16, 292-305.

Nyberg, L. and Pudas, S. (2018). Successful memory aging. Annual Review of Psychology, 70, 219-243.

Pilon, M.-H. et al. (2016). Differences in rate of cognitive decline and caregiver burden between Alzheimer's disease and vascular dementia: a retrospective study. Neurology (E-Cronicon), 2, 278.

Prince, M., Albanese, E., Guerchet, M. and Prina, $M$. (2014). World Alzheimer Report 2014: Dementia and Risk Reduction: An Analysis of Protective and Modifiable Risk Factors. London: Alzheimer Disease International.

Pudas, S., Persson, J., Josefsson, M., de Luna, X., Nilsson, L.-G. and Nyberg, L. (2013). Brain characteristics of individuals resisting age-related cognitive decline over two decades. Fournal of Neuroscience, 33, 8668-8677.

R Core Team. (2017). R: A Language and Environment for Statistical Computing. Vienna, Austria: R Foundation for Statistical Computing.

Rajan, K.B., Wilson, R.S., Weuve, J., Barnes, L.L. and Evans, D.A. (2015). Cognitive impairment 18 years before clinical diagnosis of Alzheimer disease dementia. Neurology, 85, 898-904.

Rönnlund, M., Nyberg, L., Bäckman, L. and Nilsson, L.-G. (2005). Stability, growth, and decline in adult life span development of declarative memory: cross-sectional and longitudinal data from a population-based study. Psychology and Aging, 20, 3-18.

Rouanet, A., Joly, P., Dartigues, J.F., Proust-Lima, C. and Jacqmin-Gadda, H. (2016). Joint latent class model for longitudinal data and interval-censored semicompeting events: application to dementia. Biometrics, 72, 1123-1135.

Stern, Y. (2002). What is cognitive reserve? Theory and research application of the reserve concept. Fournal of International Neuropsychological Society, 8, 448-460.

Thorvaldsson, V., Hofer, S.M., Berg, S., Skoog, I., Sacuiu, S. and Johansson, B. (2008). Onset of terminal decline in cognitive abilities in individuals without dementia. Neurology, 71, 882-887.

Wilson, R.S., Beck, T.L., Bienias, J.L. and Bennett, D.A. (2007). Terminal cognitive decline: accelerated loss of cognition in the last years of life. Psychosomatic Medicine, 69, 131-137.

Wilson, R.S., Leurgans, S.E., Boyle, P.A. and Bennett, D.A. (2011). Cognitive decline in prodromal Alzheimer disease and mild cognitive impairment. Archives of Neurology, 68, 351-356.

Zahodne, L.B. et al. (2015) Late-life memory trajectories in relation to incident dementia and regional brain atrophy. Fournal of Neurology, 262, 2484-2490. 